\title{
(2) OPEN ACCESS \\ Hormonal contraception and HIV acquisition among women: an updated systematic review
}

\author{
Kathryn M Curtis (D) , ${ }^{1}$ Philip C Hannaford (D) , ${ }^{2}$ Maria Isabel Rodriguez, ${ }^{3}$ \\ Tsungai Chipato, ${ }^{4}$ Petrus S Steyn, ${ }^{5}$ James N Kiarie ${ }^{5}$
}

\begin{abstract}
- Additional material is published online only. To view please visit the journal online (http://dx.doi.org/10.1136/ bmjsrh-2019-200509).

'Division of Reproductive Health, Centers for Disease Control and Prevention, Atlanta, Georgia, USA

${ }^{2}$ Academic Primary Care, Institute of Applied Health Sciences, University of Aberdeen, Aberdeen, UK

${ }^{3}$ Obstetrics and Gynecology, Oregon Health and Science University, Portland, Oregon, USA ${ }^{4}$ University of Zimbabwe College of Health Sciences, Harare, Zimbabwe

${ }^{5}$ Department of Reproductive Health and Research, World Health Organization, Geneva, Switzerland
\end{abstract}

\section{Correspondence to} Dr Kathryn M Curtis, Division of Reproductive Health, Centers for Disease Control and Prevention, Atlanta, GA 30341, USA; kmc6@ cdc.gov

Received 17 October 2019 Revised 3 December 2019 Accepted 4 December 2019

\section{Sinked}

- http://dx.doi.org/10.1136/ bmjsrh-2019-200557

Check for updates

(C) Author(s) (or their employer(s)) 2020. Re-use permitted under CC BY-NC. No commercial re-use. See rights and permissions. Published by BMJ.

To cite: Curtis KM,

Hannaford PC, Rodriguez MI, et al. BMJ Sex Reprod Health 2020;46:8-16.

\section{ABSTRACT \\ Objective To update a 2016 systematic review on hormonal contraception use and HIV acquisition.}

Methods We searched Pubmed and Embase between 15 January 2016 and 26 June 2019 for longitudinal studies comparing incident HIV infection among women using a hormonal contraceptive method and either non-users or users of another specific hormonal contraceptive method. We extracted information from newly identified studies, assessed study quality, and updated forest plots and meta-analyses. Results In addition to 31 previously included studies, five more were identified; three provided higher quality evidence. A randomised clinical trial (RCT) found no statistically significant differences in HIV risk among users of intramuscular depot medroxyprogesterone acetate (DMPA-IM), levonorgestrel implant (LNG implant) or the copper intrauterine device (Cu-IUD). An observational study found no statistically significant differences in HIV risk among women using DMPA, norethisterone enanthate (NET-EN), implants (type not specified) or Cu-IUD. Updated results from a previously included observational study continued to find a statistically significant increased HIV risk with oral contraceptives and DMPA compared with no contraceptive use, and found no association between LNG implant and HIV risk.

Conclusions High-quality RCT data comparing use of DMPA, LNG implant and Cu-IUD does not support previous concerns from observational studies that DMPA-IM use increases the risk of HIV acquisition. Use of other hormonal contraceptive methods (oral contraceptives, NET-EN and implants) is not associated with an increased risk of HIV acquisition.

\section{INTRODUCTION}

Observational epidemiological studies since 1991 have provided inconsistent

\section{Key messages}

- Previous data from observational studies provided inconsistent evidence about the association between depot medroxyprogesterone acetate (DMPA) and HIV acquisition.

- New data from a high-quality randomised clinical trial found no statistically significant differences in HIV acquisition among DMPA users compared with implant users or copper intrauterine device users.

- Observational data continue to suggest no association between other hormonal contraception methods and HIV acquisition.

- The World Health Organization has updated recommendations on contraceptive use for women at high risk of HIV, based in part on this new evidence.

evidence about a link between hormonal contraception use and HIV acquisition among women. ${ }^{12}$ Of particular concern has been the potential for increased risk of HIV acquisition associated with injectable depot medroxyprogesterone acetate (DMPA), a widely used contraceptive method in some areas of the world where HIV incidence rates, and maternal morbidity and mortality rates, are high. ${ }^{2}{ }^{3}$ The World Health Organization (WHO) has held several consultations to review accumulating evidence and inform recommendations for safe contraception use among women at high risk of HIV, published in WHO's Medical Eligibility Criteria for Contraceptive Use (WHOMEC). ${ }^{4}$ In 2016, a systematic review of epidemiological evidence on the association between hormonal 
contraception use and HIV acquisition was conducted in preparation for a WHO consultation. ${ }^{2}$ That review included relevant evidence published to 15 January 2016 from 31 observational studies. Most studies were considered "unlikely to inform the primary question" due to methodological limitations, and the review's appraisal focused on 14 higher-quality studies deemed "informative but with important limitations". The systematic review concluded that the evidence for most hormonal contraceptive methods did not suggest an association with HIV acquisition, but that new information had increased concerns about a possible association between DMPA and HIV risk. A key uncertainty when interpreting the findings of these observational data was the possibility of unmeasured, residual confounding distorting the true association between DMPA use and HIV acquisition, particularly in relation to condom use and other sexual behaviours.

In June 2019, results from the Evidence for Contraceptive Options and HIV Outcomes (ECHO) trial were published. ${ }^{5}$ The ECHO trial was a randomised clinical trial (RCT) specifically designed to compare HIV incidence among users of three common contraceptives: intramuscular DMPA (DMPA-IM), levonorgestrel implant (LNG implant) and the copper intrauterine device (Cu-IUD), with the goal of minimising confounding through randomisation. We provide an update of the previous systematic review, including results from the ECHO trial and other new epidemiological evidence reported since the last publication.

\section{METHODS}

This systematic review adhered to the Preferred Reporting Items for Systematic Reviews and MetaAnalysis (PRISMA) guidelines. ${ }^{6}$ We followed the same methodology that was used in the previous systematic review, ${ }^{2}$ except where noted below. The review question was: "Among women at risk of HIV, does use of a hormonal contraceptive method compared with non-use of a hormonal contraceptive method (or use of another specific hormonal contraceptive method) increase the risk of HIV acquisition?".

\section{Inclusion and exclusion criteria}

We included studies of women of reproductive age at risk of HIV infection (ie, HIV-seronegative at baseline) that compared incident, laboratory-confirmed HIV infection among women using a hormonal contraceptive method (all types of injectable, oral, implant, patch, ring, or the levonorgestrel-containing intrauterine device (LNG-IUD)), compared with women using a non-hormonal method (eg, condom, non-hormonal IUD, sterilisation, withdrawal) or no contraception. We also included studies comparing incident HIV infection among users of one specific hormonal contraceptive method compared with another specific hormonal contraceptive method (eg, DMPA users compared with norethisterone enanthate (NET-EN) users). We included longitudinal studies (observational studies, randomised trials, or meta-analyses containing data not previously published). Given the timing and location of the studies, we assumed that most, if not all, DMPA use in these studies was DMPA-IM rather than subcutaneous DMPA (DMPA-SC).

\section{Search strategy}

We searched Pubmed and Embase for the dates 15 January 2016 (the cut-off date for the last systematic review) to 26 June 2019 (online supplementary appendix 1). We hand-searched reference lists of included studies. Three authors (TC, KC, PH) screened the abstracts and full-text articles using Covidence, ${ }^{7}$ and conflicts were resolved through discussion.

\section{Data extraction and quality assessment}

We used a data abstraction form to abstract data from any newly identified studies. The previous review used a study quality assessment framework that focused on observational studies. ${ }^{2}$ Briefly, studies that failed to adjust for condom use or that had unclear measurement of hormonal contraception exposure were considered "unlikely to inform the primary question"; all other studies were considered "informative but with important limitations" to emphasise the potential for residual or uncontrolled confounding in these observational studies. For this review, we expanded the previous framework to include criteria for assessing RCTs and to define criteria for an additional higherquality level of studies that were "informative with few limitations" (online supplementary appendix 2 ). ${ }^{8}$

\section{Graphical summaries and meta-analysis}

We updated the forest plots with all newly identified reports that were considered "informative but with important limitations" or "informative with few limitations". The forest plots present adjusted point estimates and confidence intervals (CIs) for each contraceptive method compared with a non-hormonal method or no contraception, or head-to-head comparisons between two specific hormonal methods. For reports in which authors presented estimates from more than one model, we used the most fully adjusted estimates.

We also updated the meta-analysis of observational studies assessing the association between DMPA (compared with no hormonal contraception) and risk of HIV. We determined a priori that it was not appropriate to combine estimates from observational studies and RCTs into a single meta-analysis, given differences in potential sources of bias. ${ }^{9}$ Meta-analyses were conducted in Stata version 17 (StataCorp, College Station, TX, USA). We included the most fully adjusted Cox hazards ratio (HR) estimates from each study. These point estimates and 95\% CIs were log-transformed to calculate standard errors, using a random effects model. ${ }^{10}$ We evaluated statistical 
heterogeneity using the $\mathrm{I}^{2}$ statistic; a value of less than $25 \%$ was considered indicative of low heterogeneity. ${ }^{11}$

\section{Patient and public involvement}

As this analysis was based on published data, patients and the public were not involved in the design of the study.

\section{RESULTS}

\section{Description of included studies}

We screened 769 abstracts and 17 full-text reports (online supplementary figure 1). We excluded 13 fulltext reports - four reported on data already included in the 2016 review, six were letters or conference abstracts, and three had an irrelevant exposure, outcome or comparison group. ${ }^{12-24}$ From a conference abstract, ${ }^{13}$ we identified an additional 'in press' study, which has now been published. ${ }^{25}$ Therefore, five new reports met our inclusion criteria (online supplementary table 1). ${ }^{525-28}$ Two reports provided the first data from RCTs, ${ }^{5} 26$ another provided data from a new observational analysis, ${ }^{25}$ and two provided additional analyses from previously published observational studies. ${ }^{27} 28$ Two RCTs ${ }^{5} 26$ and two observational studies ${ }^{25}$ provided direct estimates for the association between hormonal contraception and HIV acquisition, while three studies (one RCT and two observational) examined various factors that might modify any association between hormonal contraceptive use and HIV risk. ${ }^{52728}$ One RCT was considered "informative with few limitations", 5 and the other "unlikely to inform the primary question". ${ }^{26}$ The three observational studies were rated "informative but with important limitations". 252728 Therefore, three new reports (from one RCT and two observational studies) provided higher-quality (either "informative with few limitations" or "informative but with important limitations") direct evidence about the association between hormonal contraceptive use and HIV acquisition. 52528

\section{Hormonal contraception and risk of HIV infection: new RCTs}

The ECHO trial provided the first evidence from an RCT that was specifically designed to compare HIV incidence rates among women using DMPA-IM, LNG implants or Cu-IUDs. ${ }^{5}$ This study did not include a group using no contraception. Investigators randomised 7829 women from 12 sites in four African countries to the three contraceptive groups. Participants were aged 16-35 years, HIV-seronegative, and seeking contraception. Follow-up visits occurred every 3 months for up to 18 months and included HIV testing, contraceptive counselling, behavioural assessment, and HIV prevention services. Modified intention-to-treat analyses were conducted, along with pre-specified analyses restricted to participants with continuous use of the assigned contraceptive method and adjusted for potential confounders including condom use and sexual behaviours. Ninety-nine percent of women accepted their assigned method, and participants used their assigned method for $92 \%$ of the follow-up time. More than $91 \%$ of participants attended each scheduled follow-up visit, 99\% had at least one follow-up HIV test, and the study accumulated 10409 woman-years of follow-up. Overall HIV incidence was 3.81 per 100 woman-years. No statistically significant differences in HIV incidence were observed among the three contraceptive groups in the main modified intention-to-treat analysis: HR 1.04 (96\% CI 0.82 to 1.33) for DMPA-IM compared with Cu-IUD; HR 1.23 (96\% CI 0.95 to 1.59) for DMPA-IM compared with LNG implant, and HR 1.18 (96\% CI 0.91 to 1.53 ) for Cu-IUD compared with LNG implant. The continuous contraceptive use analysis, which adjusted for baseline and time-varying covariates, yielded similar results. The ECHO trial was considered to be "informative with few limitations" because of robust randomisation and allocation procedures, high follow-up and contraceptive continuation rates, assessment of condom use and sexual behaviour as possible confounders, and appropriate intentionto-treat and pre-specified sensitivity analyses. Women and providers were not blinded to contraceptive group allocation; however, study personnel involved in HIV testing were blinded to contraception assignment, and the study team worked to ensure that counselling messages were consistent across the three groups.

Another open-label RCT was initiated in 2009 but stopped early because of plans to conduct the ECHO trial. ${ }^{26}$ This trial randomised women in South Africa to receive a $\mathrm{Cu}$-IUD or progestogen-only injectable (mostly DMPA, some NET-EN). ${ }^{26}$ Follow-up was planned for 12 months. The analysis included 656 injectable users and $634 \mathrm{Cu}$-IUD users who had a follow-up HIV test. All of the results presented were unadjusted. No increase in HIV seroconversion was observed between injectable users and Cu-IUD users in the intent-to-treat analysis (risk ratio (RR) 0.88, $95 \% \mathrm{CI} 0.48$ to 1.59 ) or in the per-protocol analysis (RR 0.94, 95\% CI 0.52 to 1.71). Separate per-protocol risk estimates for DMPA and NET-EN, each compared with $\mathrm{Cu}-\mathrm{IUD}$, also indicated no association. Although this study used appropriate procedures for randomisation and allocation concealment, it was considered "unlikely to inform the primary question" because of no measurement of condom use, failure to include a time-varying analysis of hormonal contraceptive exposure or description of contraceptive discontinuation or switching, and the long inter-survey interval for assessing HIV seroconversion (median 19-20 months).

\section{Hormonal contraception and risk of HIV infection: new observational studies}

A secondary analysis of an RCT of the dapivirine ring to prevent HIV assessed HIV incidence among 1136 South African study participants using DMPA-IM, NET-EN, implants (type not specified) or Cu-IUDs. ${ }^{25}$ Contraceptive use, sexual behaviour and other 
covariates, and HIV infection status were assessed during monthly visits. Ninety-five participants seroconverted over 1771 woman-years of follow-up. In contrast to previous observational studies in which the non-hormonal contraceptive comparison group included a mix of non-hormonal contraceptive use and no contraceptive use, this study used Cu-IUD users as the non-hormonal contraceptive referent group. Overall, there were no statistically significant differences in HIV incidence among users of the different contraceptive methods. When $\mathrm{Cu}$-IUD users formed the referent group, the adjusted hazards ratio (adj HR) was 0.91 (95\% CI 0.44 to 1.87) for DMPA-IM, adj HR 1.02 (95\% CI 0.49 to 2.12) for NET-EN, adj HR 0.46 (95\% CI 0.13 to 1.70 ) for implants and adj HR 0.90 (95\% CI 0.45 to 1.76 ) for all three hormonal methods considered together. When NET-EN users formed the referent group, the adj HR was 0.89 (95\% CI 0.55 to 1.44) for DMPA-IM, adj HR 0.45 (95\% CI 0.13 to 1.53$)$ for implants and adj HR 0.98 (95\% CI 0.47 to 2.03 ) for Cu-IUD. This study was considered "informative but with important limitations". Strengths included time-varying analysis of hormonal contraception exposure, condom use, and other relevant confounders; control for trial allocation group (dapivirine or placebo); clear exposure and comparison groups; and a short inter-survey interval (monthly). Limitations included no information on implant type (levonorgestrel or etonogestrel), no information about attrition within each contraceptive group, and the potential for residual or unmeasured confounding.

Another paper provided additional analyses with more participants and further follow-up time from the Mombasa Cohort, a long-term observational study of HIV acquisition among female sex workers in Kenya. ${ }^{28}$ Previous analyses had observed that both oral contraceptives (type not specified) and DMPA were associated with an increased HIV risk compared with no contraceptive use. ${ }^{29}$ LNG implant use was associated with an elevated but not statistically significant relative risk. $^{30}$ The new analysis included 1985 women with 7127 woman-years of follow-up; hormonal contraception, HIV, and relevant confounders were measured monthly. Results for oral contraceptives (adj HR 1.48, 95\% CI 1.05 to 2.09 ) and DMPA (adj HR 1.72, 95\% CI 1.34 to 2.20 ), both compared with no contraception, were almost identical to the previous results. LNG implants were not associated with HIV seroconversion (adj HR 0.99, 95\% CI 0.40 to 2.45). This study was considered "informative with important limitations", primarily because of the potential for unmeasured or residual confounding.

\section{Hormonal contraceptive use versus non-use in studies considered "informative but with important limitations" or "informative with few limitations"}

Previously, we included 31 observational studies, and focused on 14 observational studies considered "informative but with important limitations". ${ }^{2}$ Results from three newly identified publications were of sufficient quality to be added to the forest plots from the previous review (figures 1-3 and online supplementary figure 2). ${ }^{52528}$ A new estimate for the head-to-head comparison between DMPA-IM and NET-EN use was less than unity and not statistically significant, ${ }^{25}$ unlike the statistically significant, above unity, estimates from two studies in the previous review; the $95 \%$ CIs for all three estimates overlapped (figure 4). Finally, new head-to-head estimates for implants versus NET-EN and DMPA versus LNG implants were added (figure 4). The revised meta-analysis of observational studies of DMPA compared with non-hormonal or no contraception and risk of HIV acquisition resulted in a pooled OR of 1.40 (95\% CI 1.24 to 1.58 ) with an $I^{2}$ of $0 \%$, indicating low heterogeneity (online supplementary figure 3); this was almost identical to the pooled estimate in the 2016 review. $^{2}$

\section{Effect modification}

The previous systematic review did not identify consistent factors that modified any association between hormonal contraception and HIV acquisition (eg, age, herpes simplex virus type 2 (HSV-2), study site). ${ }^{2}$ New data from the ECHO trial found that age and HSV-2 status did not modify results comparing HIV incidence among the different contraceptive methods. ${ }^{5}$

Two analyses newly assessed bacterial vaginosis (BV) as an effect modifier. A cohort study in $\mathrm{Zambia}^{27}$ had previously reported no statistically significant associations for oral contraceptives, DMPA or LNG implant use with HIV acquisition. ${ }^{31}$ The new analysis examined whether the relationship between hormonal contraception and HIV acquisition was modified by a BV diagnosis among 564 women in serodiscordant couples. In the time-varying analysis, both DMPA (adj HR $6.55,95 \%$ CI 1.14 to 37.77 ) and oral contraceptives (adj HR 5.20, 95\% CI 1.68 to 16.06) were associated with HIV seroconversion during periods when BV was diagnosed, each compared with non-hormonal or no contraceptive use. No statistically significant associations were observed for DMPA (adj HR 1.35, 95\% CI 0.64 to 2.85 ) or oral contraceptives (adj HR $1.36,95 \%$ CI 0.76 to 2.42 ) among women not diagnosed with BV. Results were similar when variables for sperm on wet-prep and condomless sex in the past 3 months were added to the model. No incident HIV infections occurred among LNG implant users.

In contrast, the updated analysis from the Mombasa Cohort Study did not find that BV was an effect modifier. ${ }^{28}$ DMPA use was associated with increased risk of HIV among women with BV (adj HR 1.56, 95\% CI 1.08 to 2.25 ) and without BV (adj HR 2.08, 1.462.97), each compared with no contraceptive use. Oral contraceptive users had an elevated but not statistically significant relative risk among women with BV (adj HR 1.50, 95\% CI 0.94 to 2.39) and without BV (adj HR 
OBSERVATIONAL STUDIES

Heffron et al (2012)

Sabo et al (2019)

Morrison et al (2015) subanalysis

Morrison et al (2007/2010)

Crook et al (2014)

Wall et al (2015)

McCoy et al (2013)

Morrison et al (2012)

Balkus et al (2016)

Reid et al (2010)

Palanee-Phillips et al (2019)

Myer et al (2007)

Kleinschmidt et al (2007)

RANDOMIZED CLINICAL TRIAL

ECHO (2019) Modified ITT
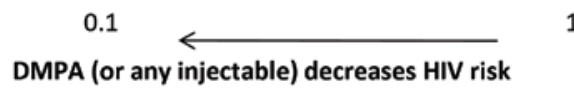

$\underset{\text { DMPA (or any injectable) increases HIV risk }}{\longrightarrow}$

adjHR injectables: 2.04 (1.03-4.04)*+

adjHR DMPA: $1.72(1.34-2.20)^{* 1}$

adjHR DMPA: 1.69 (1.02-2.78)*^

MSM adjHR DMPA: $1.48(1.02-2.15)^{*} ¥$

Cox adjHR DMPA: $1.25(0.89-1.78) ¥$

adjHR DMPA: 1.45 (1.09-1.93)*

adjHR DMPA: 1.34 (0.85-2.12)

adjHR DMPA: 1.28 (0.90-1.82)§

adjHR DMPA: 1.27 (0.93-1.73)

adjHR injectables: 1.17 (0.70-1.96)

adjHR injectables: 0.94 (0.46-1.92)

adjHR DMPA: 0.91 (0.44-1.87)

adjHR DMPA: 0.75 (0.33-1.68)

adjIRR DMPA: 0.46 (0.06-3.79)

HR 1.04 (96\% Cl 0.82-1.33)

Figure 1 Use of depot medroxyprogesterone acetate (DMPA) (or unspecified injectable) versus non-hormonal or no contraception and HIV acquisition, among 14 studies considered "informative but with important limitations" or "informative with few limitations". Error bars show 95\% confidence intervals $(\mathrm{Cls})$, with the exception of the ECHO trial which uses $96 \% \mathrm{Cl}$. Observational studies arranged in order of decreasing magnitude of risk estimate. Graph does not display estimates from marginal structural models, except where use of such models resulted in a different conclusions regarding statistical significance; in such cases, estimates from both models are displayed on a single line (also identified by bracket signs). Note: restricted to all data on DMPA or unspecified injectables, as estimates of unspecified injectables are likely comprised largely of DMPA. *Analysis showed significant findings at $p=0.05$. †Estimate for Cox model taken from slightly updated analysis that controlled for total number of unprotected sex acts. "Updated estimate from Baeten et al, 2007. 'Unpublished estimates from a sub-analysis of Morrison 2015 meta-analysis, restricted to pooled analysis using databases not previously used to publish estimates on hormonal contraceptive methods and HIV acquisition risk. ¥Different statistical models adjusted for slightly different confounders. $\S$ Unpublished estimates disaggregated by injectable type. adjHR, adjusted HR; adjIRR, adjusted incidence risk ratio; Cl, confidence interval; DMPA, depot medroxyprogesterone acetate; ECHO, Evidence for Contraceptive Options and HIV Outcomes Trial; HR, hazard ratio; ITT, intention-to-treat; MSM, marginal structural models.

1.61, 95\% CI 0.99 to 2.64$)$. LNG implants were not associated with HIV seroconversion in either group.

\section{DISCUSSION}

The previous 2016 systematic review concluded that new observational evidence at that time increased concern about a possible causal association between DMPA use and HIV acquisition, although there was uncertainty about the potential for unmeasured and residual confounding particularly from differential condom use or other measures of sexual behaviour. ${ }^{2}$ In this update, the addition of results from one new observational study, ${ }^{25}$ and updated results from a previously reported observational study, ${ }^{28}$ did not materially change the pattern of risk seen in the forest plots or the meta-analysis of observational studies suggesting an increased risk of HIV acquisition with DMPA use. ${ }^{2}$ Data from a new RCT, however, do not support such a conclusion. ${ }^{5}$ The ECHO trial found no statistically significant differences in HIV incidence among DMPA-IM users when compared with users of a non-hormonal method (Cu-IUDs) or LNG implant users. This trial provided high-quality, direct evidence of HIV risk among users of three contraceptive methods. The ECHO trial randomised women to contraceptive methods, had low attrition and high rates of continuation with the assigned contraceptive method, and found similar results of no association in both the main modified intention-to-treat analyses and secondary sensitivity analyses that controlled for several baseline and time-varying factors including condomless sex. Therefore, residual confounding is unlikely to have affected results from the ECHO trial, and so it provides higher-quality evidence about whether DMPA increases the risk of HIV acquisition in women than that from observational studies.

The ECHO trial observed no increased risk of HIV acquisition among LNG implant users when compared with Cu-IUD users or DMPA-IM users. ${ }^{5}$ These findings are consistent with three observational studies (two on 


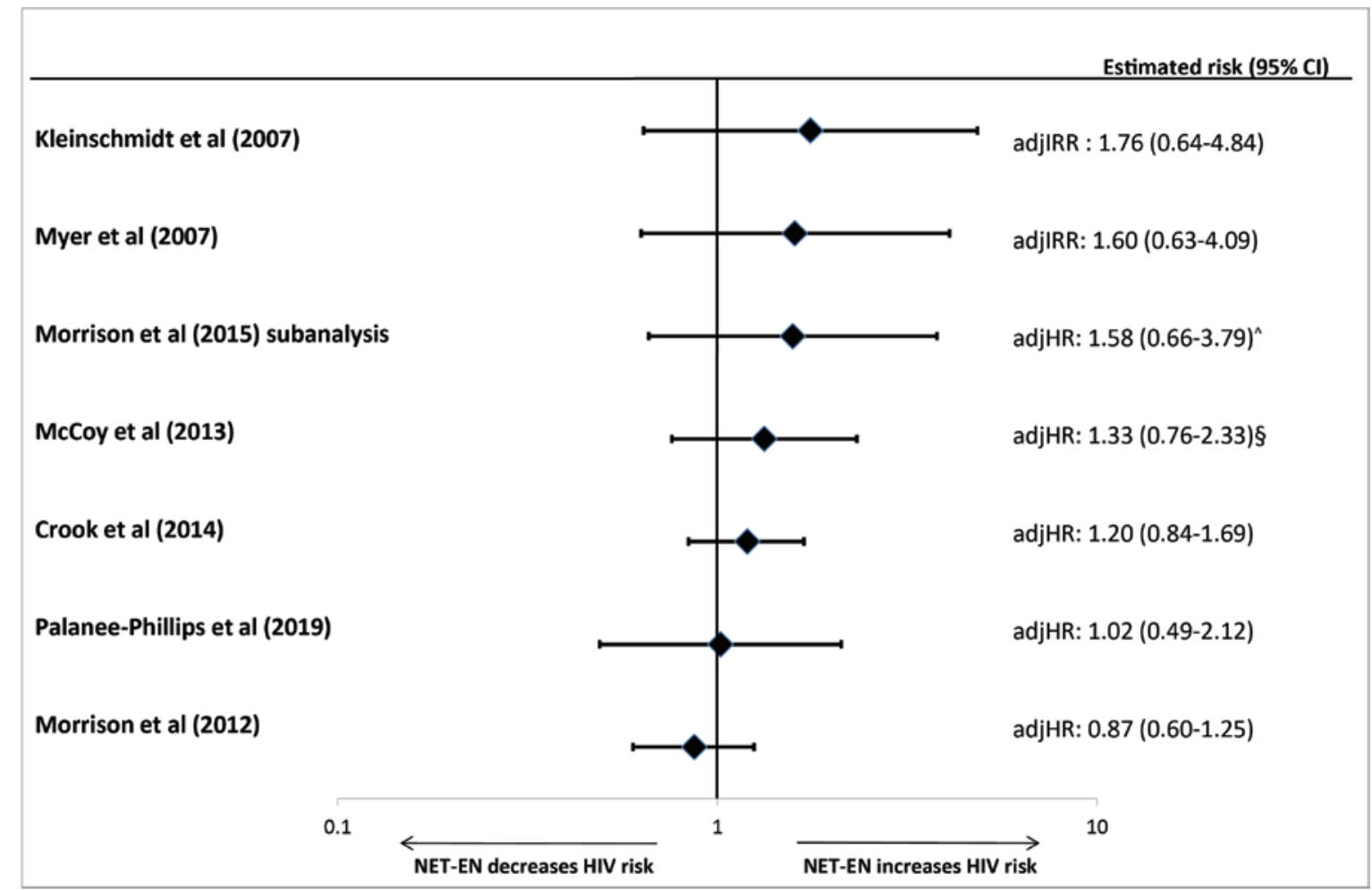

Figure 2 Use of norethisterone enanthate versus non-hormonal or no contraception and HIV acquisition, among seven studies considered informative but with important limitations. Error bars show 95\% confidence intervals. Studies arranged in order of decreasing magnitude of risk estimate. Graph does not display estimates from marginal structural models. ^unpublished estimates from a sub-analysis of Morrison et al. 2015 meta-analysis, restricted to pooled analysis using databases not previously used to publish estimates on hormonal contraceptive methods and HIV acquisition risk. §Unpublished estimates disaggregated by injectable type. adjHR, adjusted HR; adjIRR, adjusted incidence risk ratio; Cl, confidence interval; HR, hazard ratio; NET-EN, norethisterone enanthate.

LNG implants and one on unspecified implants) that found no increased risk of HIV acquisition among implant users compared with non-hormonal contraceptive users. ${ }^{25} 2811$ The study of unspecified implants also observed a statistically non-significant reduced risk of HIV among implant users compared with NET-EN users, which had not been assessed previously. ${ }^{25}$

The ECHO trial did not examine NET-EN, and therefore information on NET-EN and HIV acquisition came solely from observational studies. Previously, six studies examined HIV acquisition among NET-EN users compared with non-hormonal users, with none finding a statistically significant association; a newly identified seventh study reported an estimate consistent with those findings. ${ }^{25}$ Two observational studies had previously found an increased risk of HIV acquisition among DMPA users directly compared with NET-EN users, ${ }^{2}$ an association not observed in a newly identified study. ${ }^{25}$

The assessment of oral contraceptive use and HIV risk was also based only on evidence from observational studies. The overall body of evidence continues to suggest no increased risk of HIV among oral contraceptive users; the only new evidence was an updated estimate that was nearly identical to the previously included estimate from the same study. ${ }^{28}$
The previous review did not identify any consistent factors that modify the relationship between hormonal contraceptive use and HIV acquisition. ${ }^{2}$ Neither age nor HSV-2 status were modifiers in the ECHO trial. ${ }^{5}$ Two studies assessed for the first time whether BV is an effect modifier, ${ }^{27}{ }^{28}$ with conflicting results. Differences between the two studies included the populations studied (women in serodiscordant couples ${ }^{27}$ and female sex workers ${ }^{28}$ ), BV diagnostic techniques, measurement of potential confounders, and overall findings (ie, one study observed an association between hormonal contraception and HIV but did not find effect modification by $\mathrm{BV}^{28}$ while the study that did not find an overall association suggested effect modification). ${ }^{27}$

Although a new RCT has provided high-quality evidence about the incidence of HIV among users of two hormonal contraceptive methods, most of the body of evidence remains based on observational studies. The limitations of the observational data on this topic have been described in detail. ${ }^{2} 3233$ Key concerns are unmeasured and residual confounding, as well as unclear exposure and outcome measurement, and lack of time-varying measures in analysis. We continue to urge caution when interpreting the meta-analysis results from observational studies of DMPA and HIV acquisition, given important uncertainties about each study 


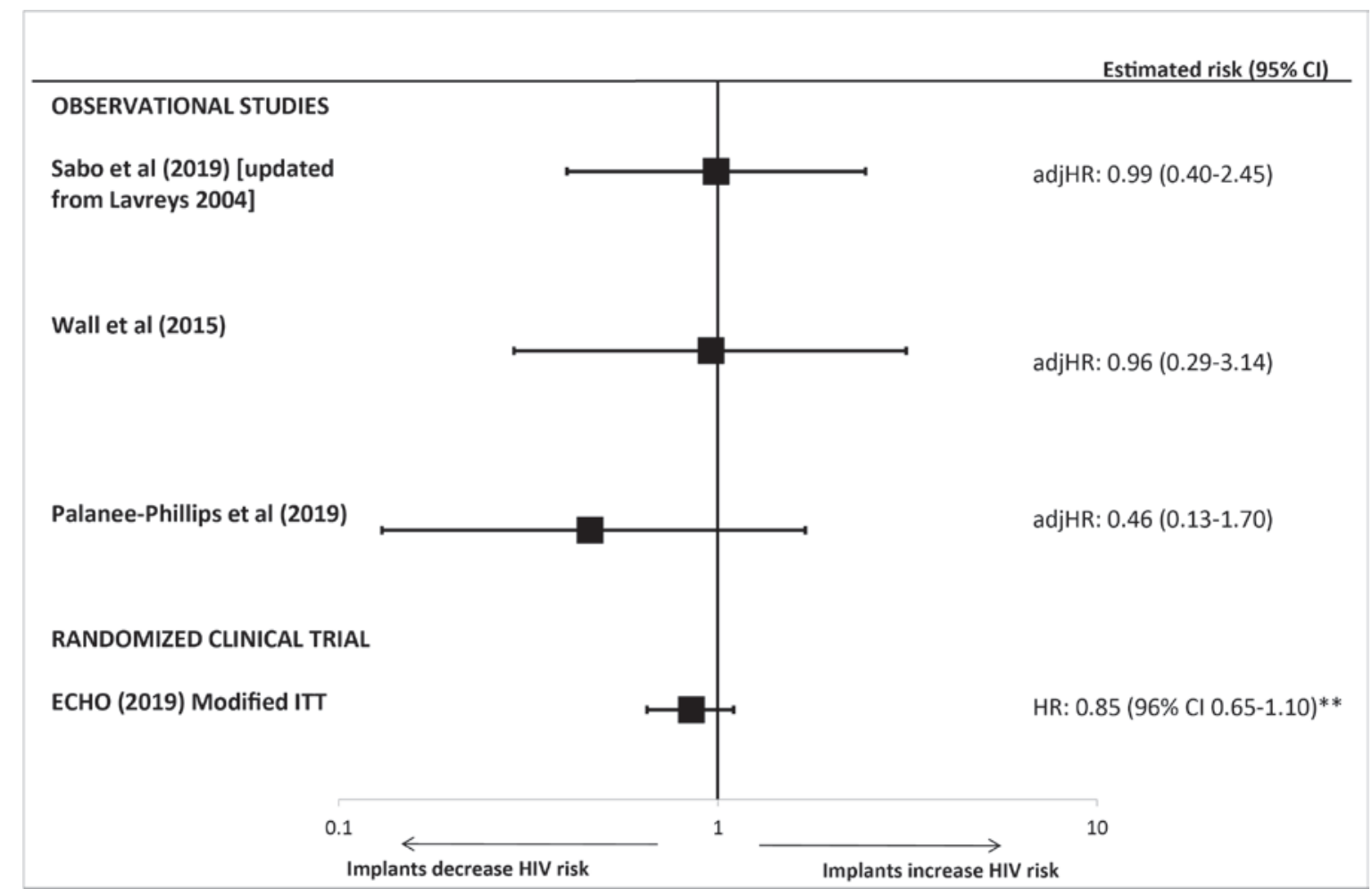

Figure 3 Use of implants* (versus non-hormonal or no contraception) and HIV acquisition, among four studies considered "informative but with important limitations" or "informative with few limitations". Error bars show 95\% confidence intervals (Cls), with the exception of the ECHO trial which uses $96 \% \mathrm{Cl}$. Studies arranged in order of decreasing magnitude of risk estimate. *Sabo et al, Wall et al and the ECHO trial included levonorgestrel implants; Palanee-Phillips et al did not specify implant type. ${ }^{* *}$ The ECHO trial reported an adjHR $1.18(96 \% \mathrm{Cl} 0.91$ to 1.53$)$ for the comparison of copper intrauterine devices (Cu-IUDs) versus levonorgestrel (LNG) implant. The inverse association is represented on this figure for LNG implant versus Cu-IUD. adjHR, adjusted $\mathrm{HR}$; Cl, confidence interval; ECHO, Evidence for Contraceptive Options and HIV Outcomes Trial; HR, hazard ratio; ITT, intention-to-treat.

included in the meta-analysis and the potential for spurious precision. For RCTs, proper randomisation should equalise confounding factors among groups at baseline. However, unblinded follow-up of participants provides opportunities for confounding and other biases to occur, for example through changes in contraceptives used or differential condom use by contraceptive group because of knowledge of the initial contraceptive allocated. Such changes need to be captured and allowed for in time-varying analyses, as was done in the ECHO trial. ${ }^{5}$ Although the ECHO trial was not designed to answer the question of whether any of the contraceptive methods assessed increased the risk of HIV acquisition when compared with no contraceptive method use, the high rates of HIV infection observed among the study groups were similar to the underlying incidence used when designing the trial. This finding provides indirect evidence of no increased risk of HIV acquisition among DMPA-IM, Cu-IUD or LNG implant users, compared with women using no contraception. Finally, unanswered questions remain about hormonal contraceptive methods for which there is currently no evidence, including DMPA-SC, etonogestrel implants, LNG-IUDs and non-oral forms of combined hormonal methods.

An earlier version of this review, which included observational studies and RCTs published before the ECHO trial, was considered during a 2019 WHO
Guideline Development Group Meeting to assess recommendations on contraception for women at high risk of HIV acquisition. WHO's updated guidance states that women at high risk of acquiring HIV infection are eligible to use all hormonal contraceptive methods and IUDs without restriction. ${ }^{34}$

\section{CONCLUSIONS}

New high-quality evidence from an RCT (the ECHO trial) did not find an increased risk of HIV among women using DMPA compared with LNG implant or $\mathrm{Cu}$-IUD users. ${ }^{5}$ These RCT data provided high-quality evidence about whether DMPA use increases HIV risk, as they are less likely to be confounded than findings from observational studies. As concluded in previous reviews, the body of evidence suggests no increased risk of HIV acquisition associated with use of other hormonal contraceptive methods.

\section{Additional educational resources}

WHO Guidance on Hormonal Contraceptive Eligibility for Women at High Risk of HIV https://www. who.int/reproductivehealth/publications/contraceptive-eligibility-women-at-high-risk-of-HIV/en/

WHO Medical Eligibility Criteria for Contraceptive Use https://www.who.int/reproductivehealth/publications/family_planning/Ex-Summ-MEC-5/en/ 


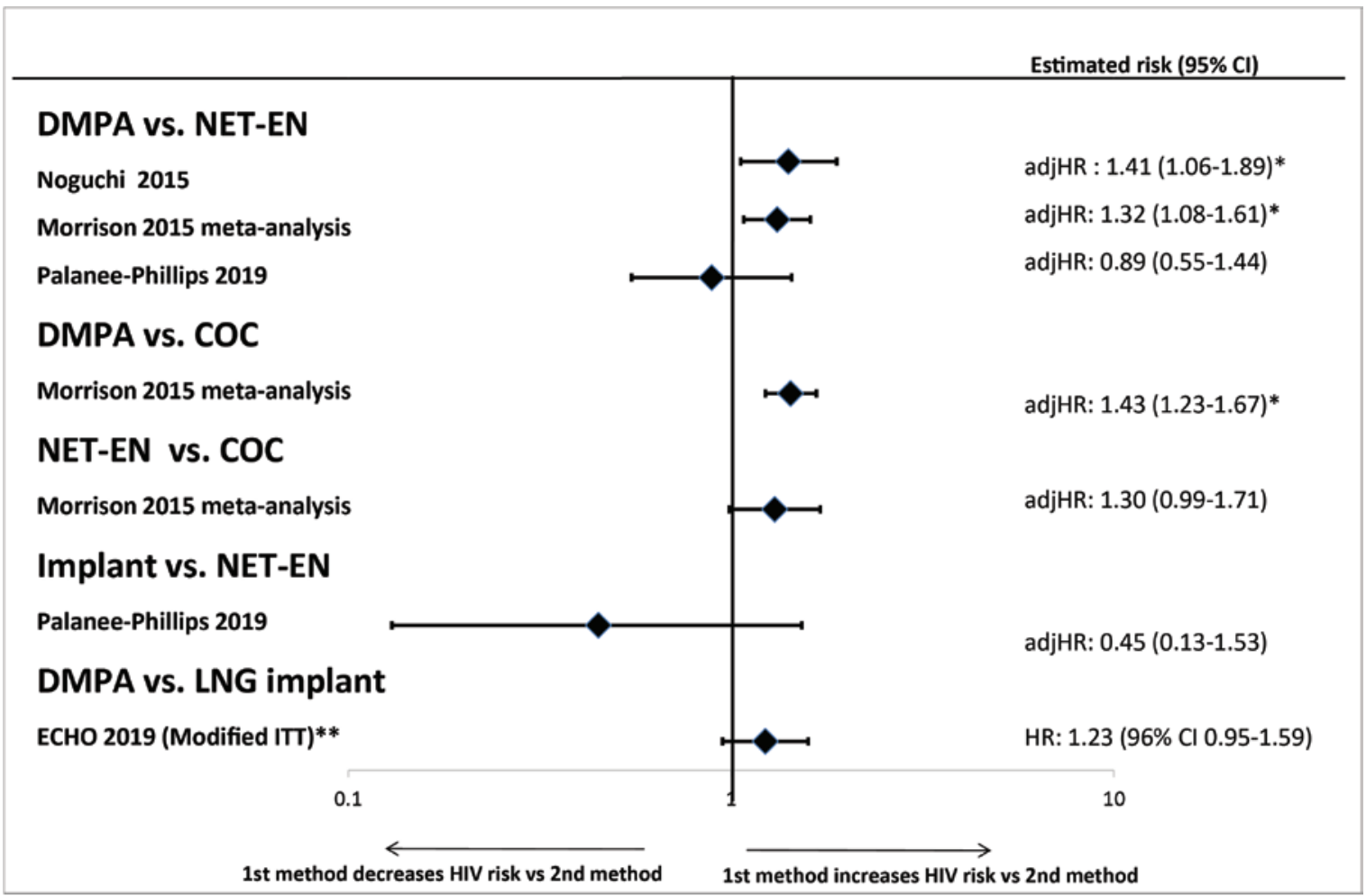

Figure 4 Hormonal contraceptive methods and HIV acquisition in head-to-head studies, among four studies considered "informative but with important limitations" or "informative with few limitations". Error bars show 95\% confidence intervals (Cls), with the exception of the ECHO trial which uses $96 \% \mathrm{Cl}$. Within each comparison group, studies are arranged in order of decreasing magnitude of risk estimate. *Analysis showed significant findings at $\mathrm{p}=0.05 .{ }^{*}$ Results from randomised clinical trial; all other results from observational studies. adjHR, adjusted $\mathrm{HR} ; \mathrm{Cl}$, confidence interval; $\mathrm{COC}$, combined oral contraception; ECHO, Evidence for Contraceptive Options and HIV Outcomes Trial; DMPA, depot medroxyprogesterone acetate; HR, hazard ratio; LNG, levonorgestrel; NET-EN, norethisterone enanthate.

WHO Selected Practice Recommendations for Contraceptive Use https://www.who.int/reproductivehealth/publications/family_planning/SPR-3/en/

Hannaford PC, Chipato T, Curtis KM. Copper intrauterine device use and HIV acquisition in women: a systematic review. BMJ Sex Reprod Health 2020;46:17-25.

Acknowledgements The authors would like to acknowledge the tremendous amount of work conducted by Chelsea Polis, $\mathrm{PhD}$, Sharon Phillips, MD, MPH, and Daniel Westreich, PhD, as authors of the 2016 systematic review on which this update is based. They would also like to acknowledge the assistance of Joanna Taliano, MA, MLS, Reference Librarian at CDC, for running the search strategies.

Contributors KC, PH, TC, PS and JK were coauthors on the previous systematic review and contributed to the planning of this updated review. $\mathrm{KC}, \mathrm{PH}$ and $\mathrm{TC}$ conducted the literature search, screening, and risk of bias assessment. MIR conducted the meta-analysis. $\mathrm{KC}$ wrote the first draft of the manuscript. All authors contributed to subsequent drafts and approved of the final manuscript.

Funding The authors have not declared a specific grant for this research from any funding agency in the public, commercial or not-for-profit sectors.

Disclaimer The findings and conclusions of this report are those of the authors and do not necessarily represent the official position of the Centers for Disease Control and Prevention, the World Health Organization, or other institutions with which the authors are affiliated.
Competing interests MIR reports personal fees from Merck (contraceptive implant trainer) and Bayer (IUD trainer), outside the submitted work. TC, JK and PS were members of the ECHO trial consortium, and JK and PS were part of the writing group for the ECHO trial results. All of the authors participated in the 2019 WHO Guideline Development Group (GDG) process which assessed recommendations on contraception for women at high risk of HIV.

Patient consent for publication Not required.

Provenance and peer review Not commissioned; externally peer reviewed.

Open access This is an open access article distributed in accordance with the Creative Commons Attribution Non Commercial (CC BY-NC 4.0) license, which permits others to distribute, remix, adapt, build upon this work noncommercially, and license their derivative works on different terms, provided the original work is properly cited, appropriate credit is given, any changes made indicated, and the use is noncommercial. See: http://creativecommons.org/licenses/by-nc/4. $0 /$.

\section{ORCID iDs}

Kathryn M Curtis http://orcid.org/0000-0002-5892-3786 Philip C Hannaford http://orcid.org/0000-0002-2588-1006

\section{REFERENCES}

1 Plummer FA, Simonsen JN, Cameron DW, et al. Cofactors in male-female sexual transmission of human immunodeficiency virus type 1. J Infect Dis 1991;163:233-9.

2 Polis CB, Curtis KM, Hannaford PC, et al. An updated systematic review of epidemiological evidence on hormonal 
contraceptive methods and HIV acquisition in women. AIDS 2016;30:2665-83.

3 World Health Organization (WHO). Hormonal contraceptive eligibility for women at high risk of HIV. Geneva, Switzerland: WHO, 2017.

4 World Health Organization (WHO). World Health Organization medical eligibility criteria for contraceptive use. 5th edn. Geneva, Switzerland: WHO, 2015.

5 Evidence for Contraceptive Options and HIV Outcomes (ECHO) Trial Consortium. HIV incidence among women using intramuscular depot medroxyprogesterone acetate, a copper intrauterine device, or a levonorgestrel implant for contraception: a randomised, multicentre, open-label trial. Lancet 2019;394:303-13.

6 Moher D, Liberati A, Tetzlaff J, et al. Preferred reporting items for systematic reviews and meta-analyses: the PRISMA statement. PLoS Med 2009; 6:e1000097.

7 Covidence Systematic Review Software. Veritas Health Information 2019.

8 Hannaford PC, Chipato T, Curtis KM. Copper intrauterine device use and HIV acquisition in women: a systematic review. BMJ Sex Reprod Health 2020;46:17-25.

9 Valentine JC, Thompson SG. Issues relating to confounding and meta-analysis when including non-randomized studies in systematic reviews on the effects of interventions. Res Syn Meth 2013;4:26-35.

10 DerSimonian R, Laird N. Meta-analysis in clinical trials. Control Clin Trials 1986;7:177-88.

11 Higgins JPT, Thompson SG, Deeks JJ. Measuring inconsistency in meta-analyses. BMJ 2003;327:557-60.

12 Baeten JM, Palanee-Phillips T, Brown ER, et al. Use of a vaginal ring containing dapivirine for HIV-1 prevention in women. Obstet Gynecol Surv 2016;71:466-8.

13 Baeten J, Palanee-Phillips T, Szydlo D, et al. Risk of HIV-1 acquisition among South African women using a variety of contraceptive methods in a prospective study. AIDS Res Hum Retroviruses 2018;34.

14 Balkus JE, Brown ER, Hillier SL, et al. Corrigendum to "Oral and injectable contraceptive use and HIV acquisition risk among women in four African countries: a secondary analysis of data from a microbicide trial" [Contraception 2016; 93 (1): 25-31]. Contraception 2016;94:95.

15 Balkus JE, Brown ER, Hillier SL, et al. Oral and injectable contraceptive use and HIV acquisition risk among women in four African countries: a secondary analysis of data from a microbicide trial. Contraception 2016;93:25-31.

16 Balkus JE, Brown ER, Palanee-Phillips T, et al. Performance of a validated risk score to predict HIV-1 acquisition among African women participating in a trial of the dapivirine vaginal ring. J Acquir Immune Defic Syndr 2018;77:e8-10.

17 Byrne EH, Anahtar MN, Cohen KE, et al. Association between injectable progestin-only contraceptives and HIV acquisition and HIV target cell frequency in the female genital tract in South African women: a prospective cohort study. Lancet Infect Dis 2016;16:441-8.

18 Haddad LB, Wall KM, Kilembe W, et al. Hormonal contraception may increase HIV acquisition among Zambian women with bacterial vaginosis. Contraception 2017;96:264.

19 Heffron R, Stalter R, Pyra M, et al. Low levels of serum medroxyprogesterone acetate (MPA) are associated with increased HIV risk. AIDS Res Hum Retroviruses 2018;34 (Supplement 1):205.

20 Heffron R, Stalter R, Pyra M, et al. HIV risk associated with serum medroxyprogesterone acetate levels among women in East and southern Africa. AIDS 2019;33:735-44.

21 Hofmeyr GJ, Lawrie TA. Authors' response to 'Comment on 'Effects of injectable progestogen contraception versus the copper intrauterine device on HIV acquisition: sub-study of a pragmatic randomised controlled trial' '. J Fam Plann Reprod Health Care 2017;43.

22 Kestelyn E, Agaba S, Van Nuil JI, et al. A randomised trial of a contraceptive vaginal ring in women at risk of HIV infection in Rwanda: safety of intermittent and continuous use. PLoS One 2018;13:e0197572.

23 Quispe Calla NE, Vicetti Miguel RD, Cherpes TL. Comment on 'Effects of injectable progestogen contraception versus the copper intrauterine device on HIV acquisition: sub-study of a pragmatic randomised controlled trial'. J Fam Plann Reprod Health Care 2017;43.

24 Wand H, Dassaye R, Reddy T, et al. Geographical-level contributions of risk factors for HIV infections using generalized additive models: results from a cohort of South African women. AIDS Care 2019;31:714-22.

25 Palanee-Phillips T, Brown ER, Szydlo D, et al. Risk of HIV-1 acquisition among South African women using a variety of contraceptive methods in a prospective study. AIDS 2019;33:1619-22.

26 Hofmeyr GJ, Singata-Madliki M, Lawrie TA, et al. Effects of injectable progestogen contraception versus the copper intrauterine device on HIV acquisition: sub-study of a pragmatic randomised controlled trial. J Fam Plann Reprod Health Care 2017;43:175-80.

27 Haddad L, Wall KM, Kilembe W, et al. Bacterial vaginosis modifies the association between hormonal contraception and HIV acquisition. AIDS 2018;32:1-604.

28 Sabo MC, Richardson BA, Lavreys L, et al. Does bacterial vaginosis modify the effect of hormonal contraception on HIV seroconversion. AIDS 2019;33:1225-30.

29 Baeten JM, Benki S, Chohan V, et al. Hormonal contraceptive use, herpes simplex virus infection, and risk of HIV-1 acquisition among Kenyan women. AIDS 2007;21:1771-7.

30 Lavreys L, Baeten JM, Martin Jr HL, et al. Hormonal contraception and risk of HIV-1 acquisition: results of a 10year prospective study. AIDS 2004;18:695-7.

31 Wall KM, Kilembe W, Vwalika B, et al. Hormonal contraception does not increase women's HIV acquisition risk in Zambian discordant couples, 1994-2012. Contraception 2015;91:480-7.

32 Polis CB, Phillips SJ, Curtis KM, et al. Hormonal contraceptive methods and risk of HIV acquisition in women: a systematic review of epidemiological evidence. Contraception 2014;90:360-90.

33 Polis CB, Westreich D, Balkus JE, et al. Assessing the effect of hormonal contraception on HIV acquisition in observational data: challenges and recommended analytic approaches. AIDS 2013;27:S35-43.

34 World Health Organization. Hormonal contraceptive eligibility for women at high risk of HIV, 2019. Available: https:// www.who.int/reproductivehealth/publications/contraceptiveeligibility-women-at-high-risk-of-HIV/en/ 